\title{
Elastic Versus Inelastic Numerical Modelling of Deep and Highly Stressed Mining Fronts
}

\author{
P.P. Andrieux Itasca Consulting Canada Inc., Canada \\ R.K. Brummer Itasca Consulting Canada Inc., Canada \\ H. Li Itasca Consulting Canada Inc., Canada \\ C.P. O’Connor Itasca Consulting Canada Inc., Canada
}

\begin{abstract}
With the current trend of exploiting mines at increasing depths comes the challenge of managing increasingly high stress conditions. One tool at the disposal of mine engineers and designers is the numerical simulation of the stresses and deformations likely to be encountered ahead of the mining fronts for various mining scenarios. Although the benefits of numerical modelling are by now well accepted throughout the industry, the applicability of the various types of numerical modelling approaches is not always well understood and still the subject of much debate.

This paper endeavours to explain the basic differences between the elastic and inelastic approaches, as well as provide practical guidelines concerning which one to use for various sets of circumstances. Case studies of high stress situations are provided that show where the simpler elastic approach was adequate, and where implementing an inelastic approach was unavoidable. Some insight is also provided into the type of practical information that can be extracted from advanced failure analyses (the exclusive domain of inelastic techniques), such as determining the degree of failure of a rock mass by examining the stress state of its failed zones, which indicates their position along the strain-softening post-peak response typical of hard rock masses.
\end{abstract}

\section{Introduction}

As underground mines tend to reach ever increasing depths, properly managing the associated ever increasing stress conditions becomes critically important. This management comes largely from strategic long-term decisions made early in the process, concerning the mining method, the size and geometry of the stopes, and the extraction sequence. The numerical simulation of the stresses and deformations that can be expected to occur in different areas at different mining steps and for various mining scenarios is a powerful tool at the disposal of mine engineers and designers to help them make such decisions. Extensive numerical modelling campaigns are nowadays commonly performed for deep projects where high stress levels are expected to develop ahead of the excavations and potentially affect mining.

The applicability of the various types of numerical modelling methods, and particularly concerning elastic (also referred to as linear) versus inelastic (non-linear) approaches, is however not always well understood and still the subject of much debate in the industry. This paper is an attempt at clarifying certain misconceptions, and at providing real-life examples of high stress situations that illustrate where the simpler elastic approach can be sufficient, and where the more complex inelastic approach is necessary. Note that all the examples shown in this paper are from real consulting projects on deep and/or highly stressed mining, which were completed using inelastic modelling - we re-ran the models elastically to provide the comparisons presented in this article. Some insight is also provided into the type of additional information inelastic modelling can provide.

\section{Elastic versus inelastic numerical modelling}

Elasticity constitutes the first response of any solid material subjected to a force, from the moment it begins to deform in response to this force, to the moment irreversible damage starts to occur in it. If the force is 
large enough all solid materials eventually fail, or "break", under the load, and their behaviour becomes much different (and, in particular, largely non-linear). Inelasticity is concerned with the description of the behaviour of a material after it has failed. These concepts of elastic and inelastic behaviours are commonly used to describe two fundamentally different approaches for numerically simulating stresses, deformations and, in the case of the inelastic approach, failure profiles in a solid material subjected to a set of forces.

\subsection{Elastic modelling}

Elastic modelling considers the material being modelled as perfectly elastic, i.e. as being able to deform indefinitely without failing as more load is applied to it. Note that elasticity works both ways: the material can be indefinitely stretched when subjected to a tensile load, and indefinitely compressed when subjected to a compressive load. Since no failure (defined as permanent damage) is allowed to occur, elastic modelling also implies a full return to the initial state when the load is removed. The relationship between load and deformation is linear and defined by the modulus of elasticity $\mathrm{E}$ (the slope of the line, also referred to as the Young's modulus) of the material, as shown in Figure 1.

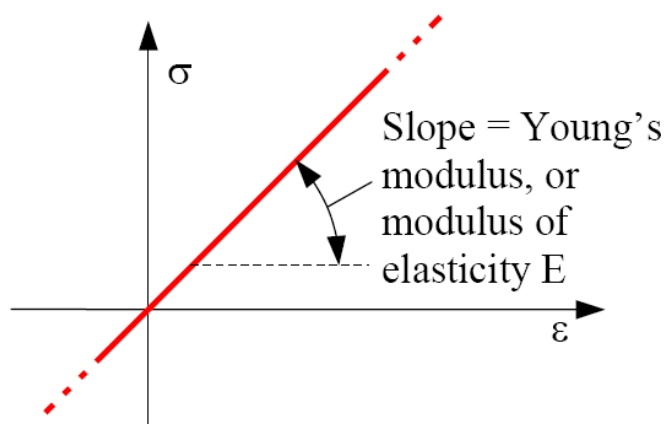

\section{Figure 1 Representation of a perfectly elastic material, and definition of the modulus of elasticity}

As can be seen in Figure 1, the modulus of elasticity, E, is defined as:

$$
\mathrm{E}=\sigma \div \varepsilon
$$

with $\sigma$ the stress applied to the material and $\varepsilon$ the strain caused in it as a result of this stress - hence, E is expressed in units of pressure. The behaviour of a perfectly elastic material is also governed by its Poisson's ratio $v$, which, depending upon whether a compressive or a tensile load is applied, is defined as either the ratio of transverse extension strain to longitudinal contraction strain (in the direction of the applied compressive force), or, the ratio of transverse contraction strain to longitudinal extension strain (in the direction of the applied tensile force). In other words, what the Poisson's ratio controls is the amount by which a sample of material that is compressed (or stretched) in one direction will get thicker (or thinner) in the other two directions.

For an isotropic material, Hooke's Law of elasticity can be generalised in the three dimensions, as follows (Assonvi \& Richter, 1979):

$$
\begin{aligned}
& \varepsilon_{\mathrm{xx}}=\left[\sigma_{\mathrm{xx}}-v\left(\sigma_{\mathrm{yy}}+\sigma_{\mathrm{zz}}\right)\right] \div \mathrm{E} \\
& \varepsilon_{\mathrm{yy}}=\left[\sigma_{\mathrm{yy}}-v\left(\sigma_{\mathrm{xx}}+\sigma_{\mathrm{zz}}\right)\right] \div \mathrm{E} \\
& \varepsilon_{\mathrm{zz}}=\left[\sigma_{\mathrm{zz}}-v\left(\sigma_{\mathrm{xx}}+\sigma_{\mathrm{yy}}\right)\right] \div \mathrm{E}
\end{aligned}
$$

with: $\varepsilon_{\mathrm{xx}}, \varepsilon_{\mathrm{yy}}$ and $\varepsilon_{\mathrm{zz}}$ the strains along the perpendicular $\mathrm{x}, \mathrm{y}$ and $\mathrm{z}$ axes, respectively; $\sigma_{\mathrm{xx}}, \sigma_{\mathrm{yy}}$ and $\sigma_{\mathrm{zz}}$ the stresses acting along the same $\mathrm{x}, \mathrm{y}$ and $\mathrm{z}$ axes, respectively; $v$ the Poisson's ratio (taken to be the same in all directions, i.e. considered isotropic); and, $\mathrm{E}$ the modulus of elasticity.

A perfectly (i.e. "endlessly", in this context) elastic behaviour is generally not physically representative of the real behaviour of rock, which is actually quite fragile - rock can in reality only sustain very small amounts of strain before yielding and starting to accumulate irreversible damage. The main attraction of considering rock in a numerical model as a perfectly elastic medium is the simplicity of the method, whereby 
1) only simple parameters (which are easily derived in the laboratory) are required, 2) the mathematical equations involved are easily implemented in a computer code and 3) the computation of stress and deformation results is relatively quick.

\subsection{Inelastic modelling}

Contrary to elastic modelling, inelastic modelling considers the solid material as being able to fail under the load. Hence, it considers that the linear elastic response is limited and followed by a non-linear post-peak response. Note that the formulation of the elastic behaviour of the material is the same with both the elastic and inelastic modelling approaches. Figure 2a illustrates the complete idealised behaviour of a sample of moderately jointed hard rock subjected to an external force and a given level of confinement. The elastic response stops once the yielding point is attained, after which the sample will start to undergo irreversible damage and (plastic) deformation. The post-peak response can itself be broken down into a number of successive phases. A brittle behaviour is usually experienced first, whereby the material's ability to bear a load will suddenly and rapidly decrease immediately after its peak strength has been attained. A softening response generally follows, whereby additional deformation (caused by the load still acting upon the sample) will cause further weakening of the material, but in a more progressive manner. Complete failure (disintegration of the sample) generally follows. Note that the extent of each successive phase of the response is dependent upon the mechanical characteristics of the material - certain materials are more brittle (glass, for example), whereas others exhibit a much more ductile behaviour (wood, for example), with a dominant softening response.

It is not practical (and not necessary, given the many other simplifications that must be made in a large-scale numerical model) to try to mimic precisely the exact behaviour of a given solid material. Instead, this behaviour is simplified, generally in the form of one of the three behaviours shown in figures $2 \mathrm{~b}, 2 \mathrm{c}$ and $2 \mathrm{~d}$.

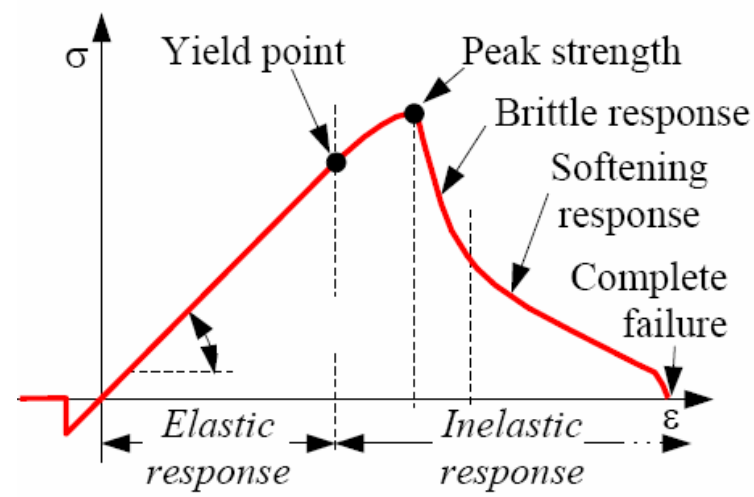

(a) Actual behaviour (idealised).

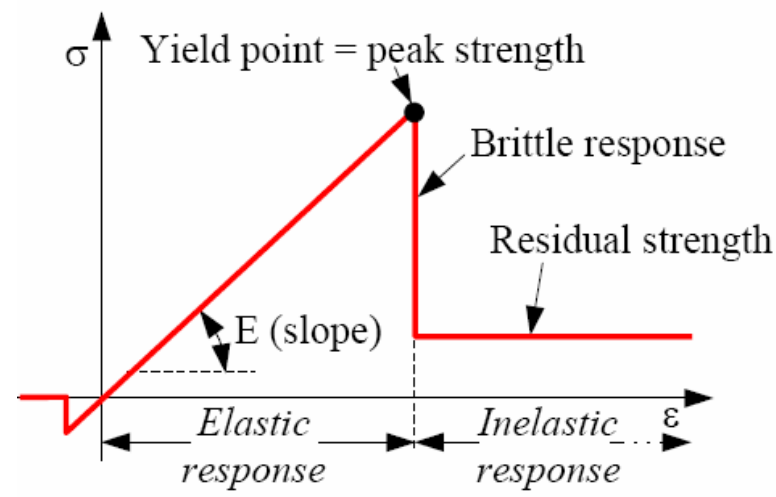

(c) Brittle representation.

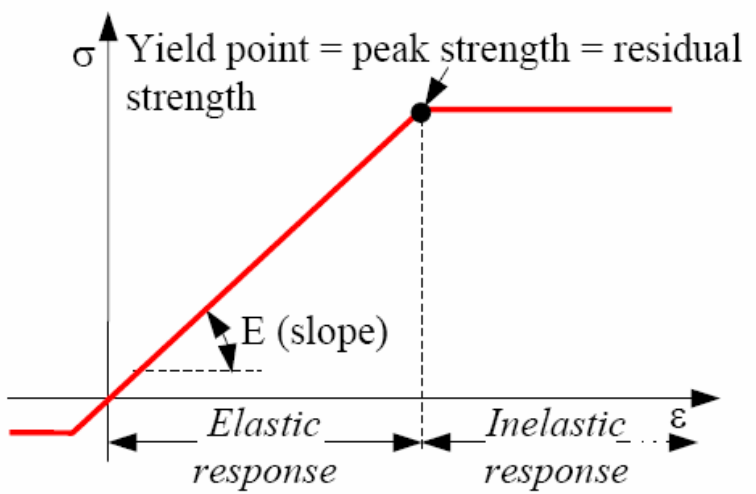

(b) Elastic-perfectly plastic representation.

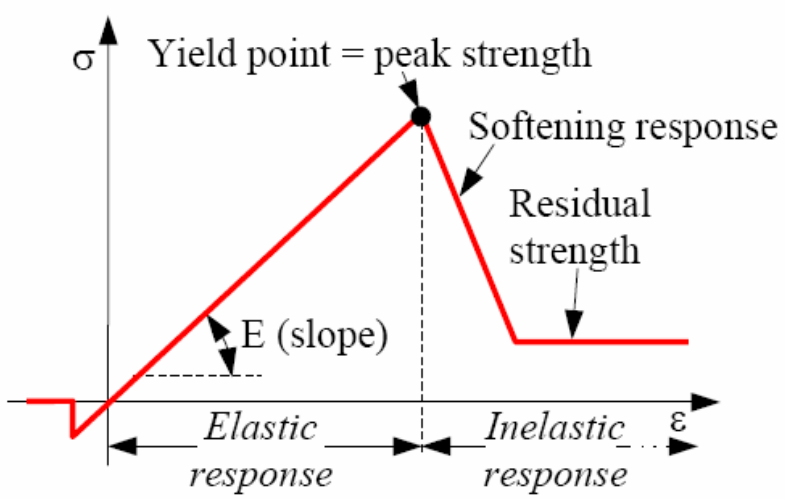

(d) Strain-softening representation.

Figure 2 Inelastic behaviour typically associated with a moderately jointed hard rock mass (a), and various common representations of this behaviour in inelastic numerical models (b), (c) and (d) 
The simplest approach is shown in Figure 2b, whereby once the rock has reached its peak strength no additional stress can be carried and perfect plastic deformation occurs under constant load (this constitutive behaviour is sometimes referred to as the "Mohr-Coulomb" model). Although this behaviour limits the amount of load the material can sustain, which is realistic, it is not overly representative of a typical moderately jointed hard rock mass, where our interest lies. The brittle strain-softening behaviour shown in Figure $2 \mathrm{c}$ represents the simplest expression of a more realistic behaviour, whereby the load-bearing capacity of the rock suddenly drops to its residual level immediately after attaining its peak level this would represent an extremely brittle behaviour, typical of very fine-grained, isotropic and homogeneous solid materials.

A strain-softening behaviour more realistic of a moderately jointed hard rock mass, whereby the brittle response of the rock mass is more progressive, is shown in Figure 2d. Note that at the scale of a laboratory sample, complete failure eventually occurs if the test is continued (as shown in Figure 2a). At the scale of the rock mass, however, even completely failed rock (i.e. rock that has reached its residual state) can retain a certain level of minimum load-bearing capacity if it is completely surrounded by solid material, does not have the kinetic freedom to completely collapse into a nearby void, and continues to occupy a certain volume. This behaviour is captured in the brittle and strain-softening models shown in Figure $2 \mathrm{c}$ and Figure $2 \mathrm{~d}$, whereby some level of residual strength is maintained, which corresponds to the compressive load the material can continue to carry "indefinitely", or, in other words, the minimum and constant level of stress it can continue to sustain at the level of confinement it is subjected to as deformation continues to increase. Brittle and strain-softening behaviours promote failure by reducing the load-bearing capacity of failed rock, which requires a more extensive stress transfer to occur before a state of equilibrium can be reached again.

Figure 3 illustrates this effect in the case of a strain-softening behaviour. Consider a conceptual $13 \mathrm{~m}$ diameter horizontal tunnel driven in rock at $90^{\circ}$ to $\sigma_{1}$ at a depth of $2000 \mathrm{~m}$ below surface. Assume that at this location $\sigma_{1}$ and $\sigma_{2}$ are horizontal, and $\sigma_{3}$ is vertical, and consider in situ magnitudes of 80,60 and $54 \mathrm{MPa}$ for $\sigma_{1}, \sigma_{2}$ and $\sigma_{3}$, respectively. Figure 3 shows cross-sections (cut at the same location along the tunnel) on which the surrounding stress field is displayed, as computed in $3 \mathrm{D}$ with a distinct element code (3DEC [Itasca, 2003] in this case), for various rock mass constitutive behaviours. Note in Figure 3c how much further away from the tunnel boundaries the high stress fronts locate with a strain-softening behaviour (taken to be that of the Sudbury Basin Granite). Note also how in the case of the elastic behaviour the high stress fronts are directly in contact with the tunnel surface.

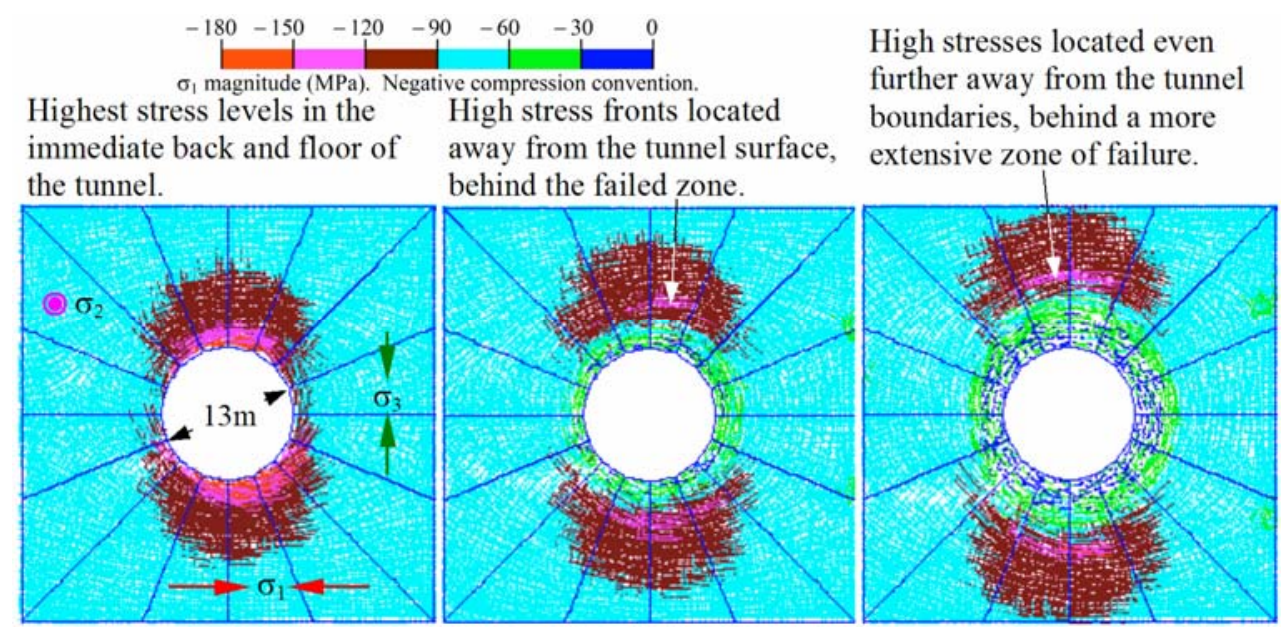

Figure 3 Cross-sectional views of the stress field around a conceptual circular tunnel $2000 \mathrm{~m}$ below surface subjected to the same in situ stress field, but sitting in a rock mass modelled with different behaviours. All stresses are at the same scale and represented by principal stress vectors colour-coded by $\sigma_{1}$ magnitude

Looking at the various graphs in Figure 2, it is clear that inelastic modelling is more complicated to conduct than elastic modelling. Firstly, more parameters are needed in order to describe the peak strength and postpeak behaviour of the material - some of these parameters are also quite difficult to establish at the scale of the rock mass (e.g. the failure criterion, the brittle and softening responses, as well as the residual strength). 
If an inelastic approach is implemented (i.e. if the rock mass is allowed to fail) and regardless of the postpeak constitutive behaviour retained, then failure criteria need to be derived, which represent the strength of the various geological units comprising the rock mass as a function of the level of confinement they are subjected to. Figure 4 shows the main failure criteria commonly used in numerical modelling applications. With the linear Mohr-Coulomb failure criterion shown in Figure 4a, strength is defined as the amount of shear stress the material can withstand at various levels of normal stress (normal to the shear plane). Equation (5) describes this failure criterion:

$$
\tau=\mathrm{c}+\left(\sigma_{\mathrm{N}} \tan \phi\right)
$$

with: $\tau$ and $\mathrm{c}$ the shear strength and cohesion of the material, respectively; $\sigma_{\mathrm{N}}$ the normal stress; and, $\phi$ the friction angle of the material (expressed in degrees), and with $\tau, \mathrm{c}$ and $\sigma_{\mathrm{N}}$ expressed in consistent units of pressure. Note also in Figure 4a the tensile cut-off at the left end of the graph.

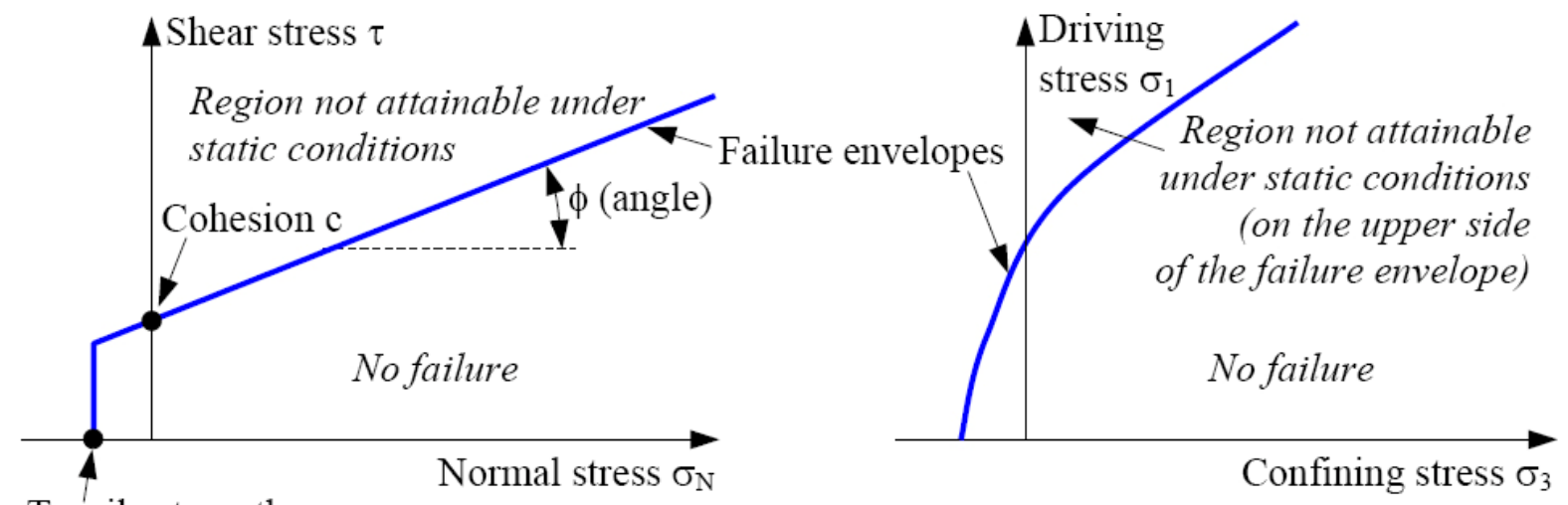

Tensile strength $\sigma_{\mathrm{T}}$

(a) Linear Mohr-Coulomb failure criterion.

(b) Non-linear Hoek-Brown failure criterion.

\section{Figure 4 Linear Mohr-Coulomb (a) and non-linear Hoek-Brown (b) failure criteria for rock}

The non-linear Hoek-Brown failure criterion (Hoek and Brown, 1980) is also widely used, whereby strength is represented by the amount of $\sigma_{1}$ driving stress the material can withstand at various levels of $\sigma_{3}$ confinement. This criterion, shown in Figure 4b, has the following general formulation:

$$
\sigma_{1}=\sigma_{3}+\sigma_{\mathrm{ci}}\left(\left[\left\{\mathrm{m} \sigma_{3}\right\} \div \sigma_{\mathrm{ci}}\right]+\mathrm{s}\right)^{a}
$$

with: $\sigma_{1}$ and $\sigma_{3}$ the major and minor principal stresses at failure, respectively; $\sigma_{\mathrm{ci}}$ the uniaxial compressive strength of the intact rock material; $\mathrm{m}$ and $\mathrm{s}$ the Hoek-Brown parameters; and, $a$ an exponent that depends upon the rock mass characteristics.

Let us now consider what happens when failure occurs. When the stress field in a volume of rock reaches the failure envelope, failure occurs, the strength of the material is reduced, and the acting stresses redistribute around this volume in order for no volume of rock to be subjected to a stress field that lies above the failure envelope. Note that as failure progresses the friction angle, cohesion and tensile strength decrease, eventually reaching their residual values, which define another strength curve that describes the strength of the failed material under various states of confinement, as illustrated by the bottom curve in Figure 5 . Intermediate strength envelopes between the intact (peak) and the completely failed (residual) states can be described by the combination of the friction angle, cohesion and tensile strength values at the corresponding intermediate levels of accumulated plastic strain. 


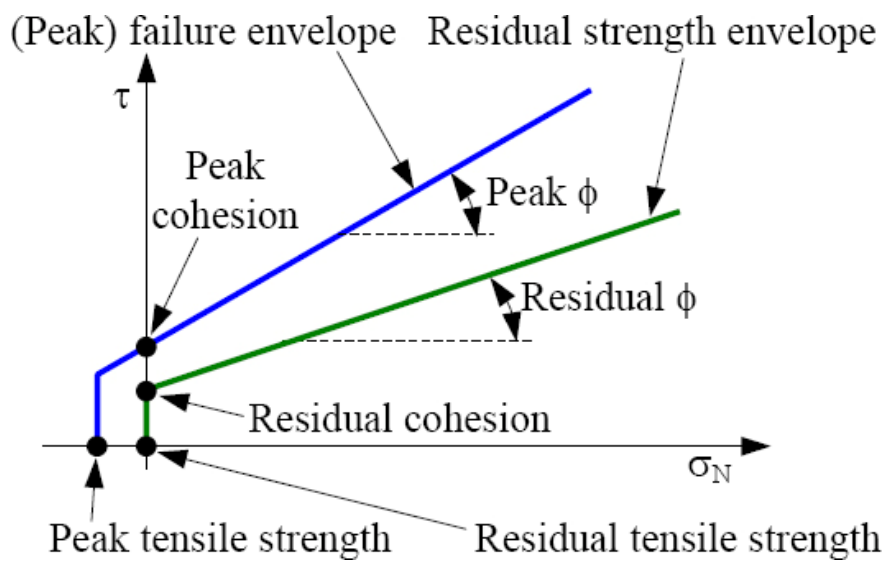

Figure 5 Mohr-Coulomb failure (top curve) and residual (bottom curve) envelopes in the $\left(\tau-\sigma_{N}\right)$ space, which describe the strength of the intact and failed material under various levels of confinement

Accurately defining all the required parameters is unarguably the single largest difficulty associated with inelastic numerical modelling - this challenge is further compounded by the inherent variability of rock as a material. The mechanical behaviour and mathematical equations involved are also more complex and not as easily implemented in a computer code. One difficulty is the need for the code to continuously track failure and redistribute the stresses accordingly throughout the model - this requirement complicates the procedure and significantly lengthens the duration of the simulations. This is however a fundamental advantage of inelastic modelling. In most deep and high stress mining situations failure does not only occur, but very much controls the mechanical behaviour of the rock mass in the vicinity of the mining fronts. As the rock fails ahead of the mining fronts, stresses will redistribute and equilibrate deeper into the rock mass, creating continuously evolving regions of high stress concentration and stress relaxation. This crucial load redistribution mechanism, which is a critical input to the design of any deep and highly-stressed mine, needs to be properly captured and predicted throughout the life of the operation - only an inelastic method can readily accomplish this.

\subsection{The “Centre of Gravity" of effort for elastic versus inelastic numerical modelling}

Effort must be focused differently with elastic and inelastic approaches when modelling high stress situations known to result in the failure of the rock mass.

\subsubsection{Centre of gravity of effort for elastic modelling}

With an elastic method there is little pre-computational effort required since the few input parameters needed are relatively straightforward and easy to obtain. However, when high stress levels are computed (higher than the rock mass can in fact sustain), substantial post-computational interpretation may be required. With deep and high stress mining the strength of the rock mass is generally expected to be exceeded, and locating the failure front ahead of mining becomes of great importance since it largely dictates where seismicity is likely to occur (along the edge of this front) and where gravity-driven failures can be expected to take place (in the relaxing regions behind this front). Under such circumstances elastic modelling can provide useful insight only if it can give some reliable indication of where the failure front is located at successive mining steps. Since this location is partly controlled by the post-peak state of the failed rock ahead of mining, which is not taken into account by elastic models, it can be difficult to derive from elastic results.

The way this is generally addressed is by conducting site-specific empirical back-analyses whereby the observed behaviour of the rock mass at certain locations is compared to the stress levels computed by the elastic model at these locations at the corresponding time steps. The end objective is to derive empirical relationships between the elastic stress magnitudes computed and specific rock mass behaviours (seismicity, gravity-driven failures, etc.), generally in the form of simple expressions, such as ( $\left.\sigma_{1}+b \sigma_{3}\right) \div c \geq d$, for example, where the constants $\mathrm{a}, \mathrm{b}, \mathrm{c}$ and $\mathrm{d}$ are fitted to some underground observations. 
Consider the example shown in Figure 6 and Figure 7, which pertains to a very deep underground metal mine in Canada. These figures show elevation views of the evolution of the major and minor principal stress magnitudes ahead of a mining front that progresses upwards towards another mining horizon at a depth of about $2 \mathrm{~km}$, as computed by the same three-dimensional distinct element code (3DEC in this case) for an inelastic strain-softening (left) and elastic (right) rock mass behaviour. In this case the inelastic model was adjusted against numerous recorded seismic events and will be taken as the reference against which the elastic results will be compared. Let us examine how the elastic results could be used to infer where the failure front is located at each successive mining step. Since with a Hoek-Brown criterion failure is dictated by the magnitude of $\sigma_{1}$ and $\sigma_{3}$ (as per Figure 4b), let us see how the elastic stresses computed could be used to estimate the $\sigma_{1}$ and $\sigma_{3}$ magnitudes computed by the strain-softening model at corresponding time steps.

At each of the three mining steps shown in Figure 6 a series of points have been drawn on the left hand side figure at locations where the inelastically computed $\sigma_{1}$ magnitude is about $120 \mathrm{MPa}$ in compression, which is taken to correspond to the approximate extent of the high $\sigma_{1}$ front ahead of mining. These points were then copied directly on top of the corresponding right hand side figure (which is at the same scale), in order to assess how the elastic stresses could have been used to infer the inelastic results. The same process was repeated in Figure 7, whereby the markers were this time located where the inelastically computed $\sigma_{3}$ magnitude is approximately $60 \mathrm{MPa}$ in compression. Note that the orebody being non-planar and of varying thickness, it is not possible to select a single viewing plane that captures the stress field everywhere on the immediate edge of the excavations.

As far as $\sigma_{1}$ is concerned (Figure 6), the $120 \mathrm{MPa}$ contours from the inelastic simulations correspond to an elastic $\sigma_{1}$ magnitude of around 80 to $100 \mathrm{MPa}$. Note however that the location of the markers on the elastic results is on top of virgin (pre-mining) magnitudes, which makes a direct estimation (based upon magnitude alone) of the corresponding inelastic results difficult. As shown in Figure 7, the elastic $\sigma_{3}$ results are even more difficult to visually "convert" to inelastic values. 

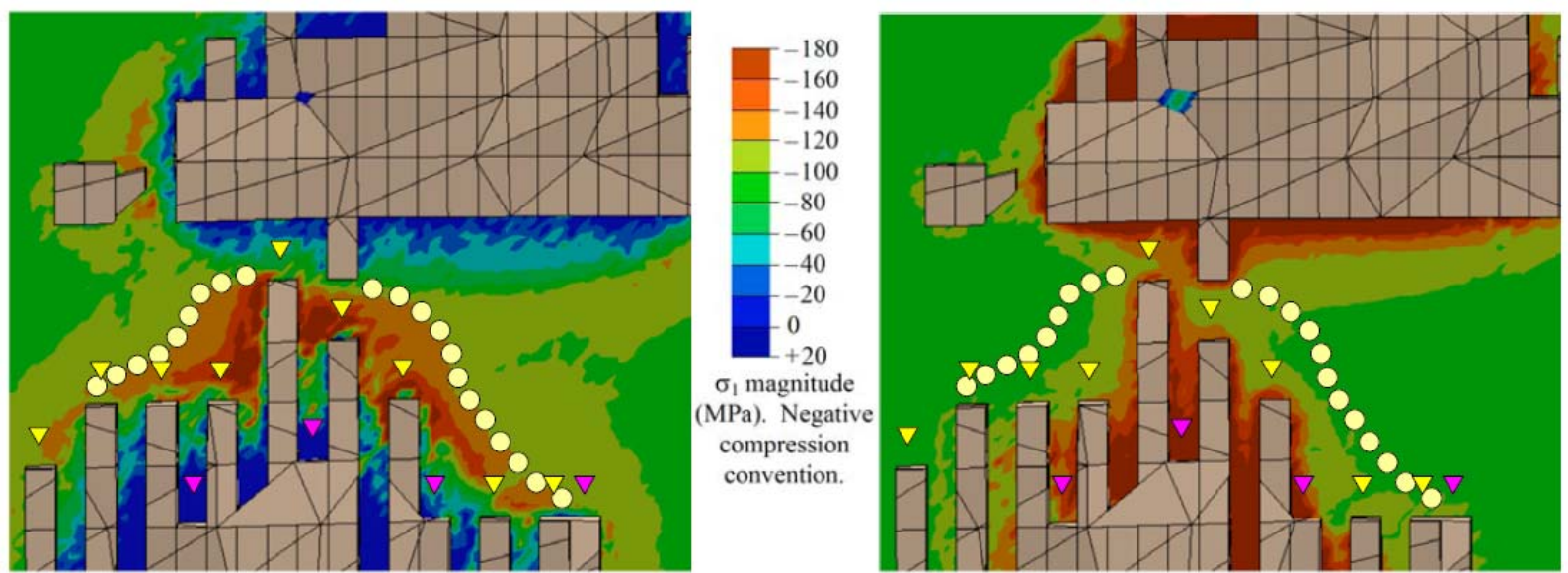

Inelastic strain-softening.

(a) At mining step $A$.

Elastic.
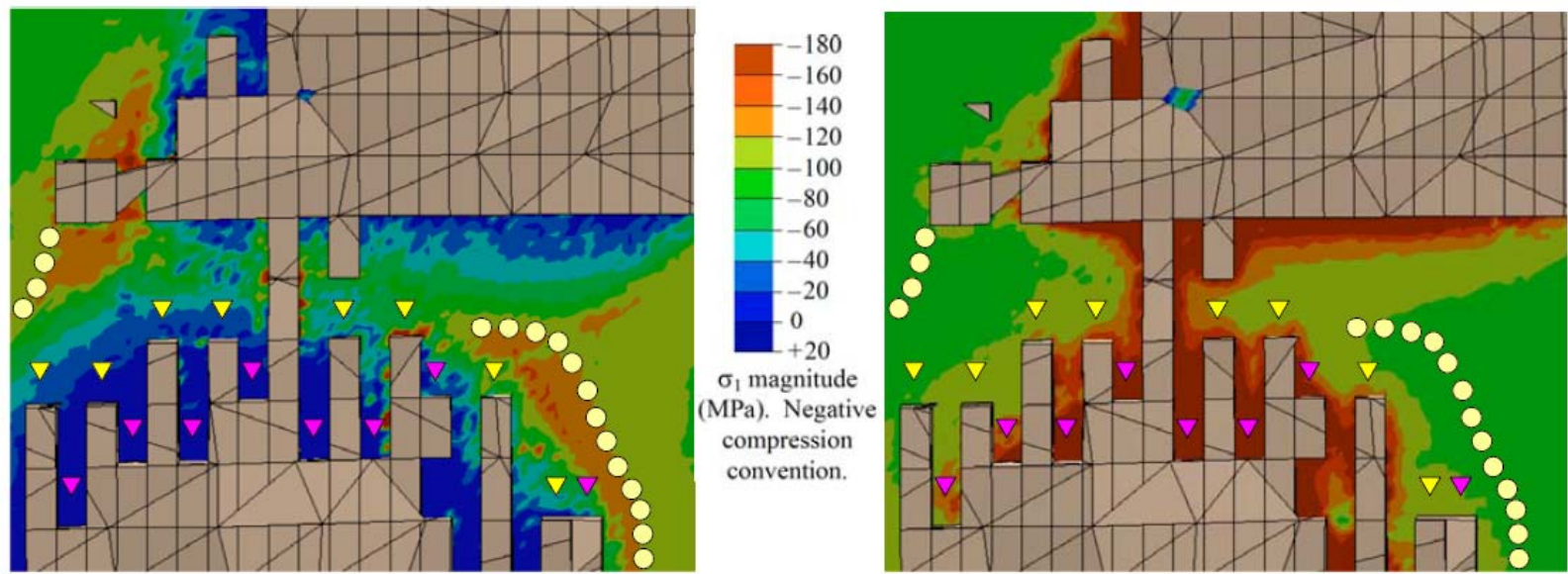

Inelastic strain-softening.

(b) At mining step $B$.

Elastic.
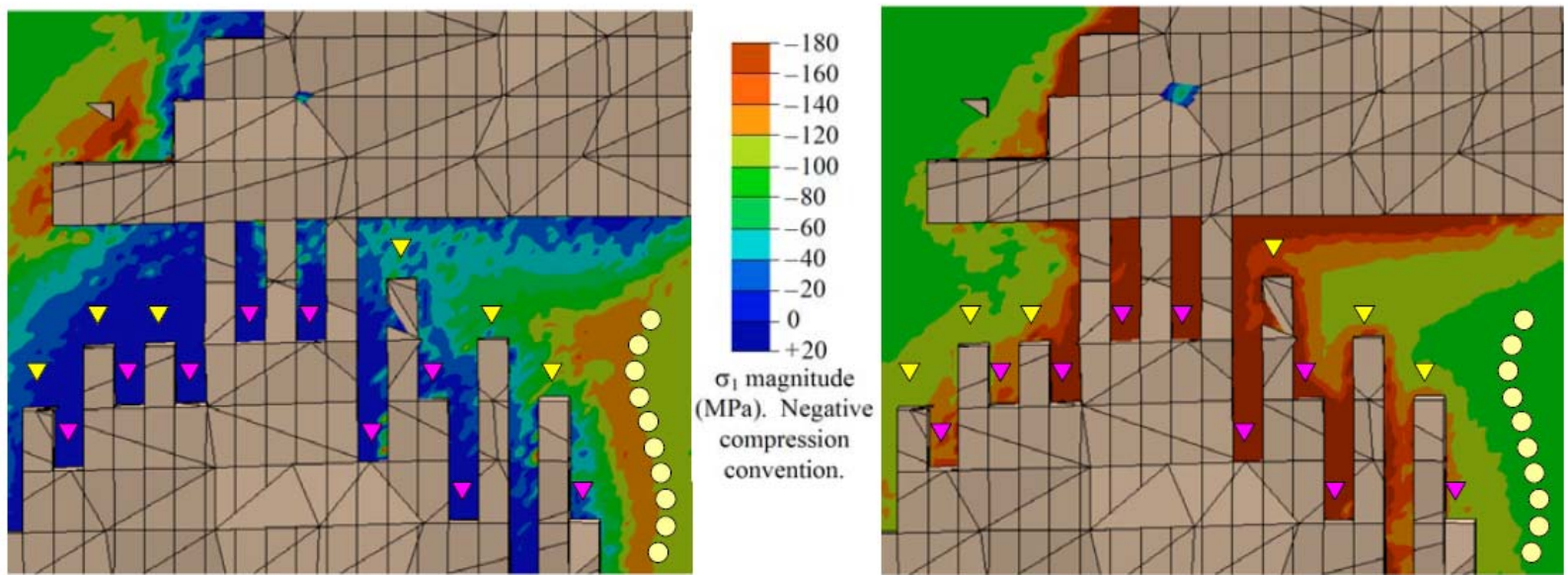

Inelastic strain-softening.

(c) At mining step $C$.

Elastic.

Figure 6 Elevation views showing the evolution of the major principal stress magnitude ahead of a mining front progressing towards another mining horizon, for an inelastic strain-softening (left) versus elastic (right) rock mass behaviour

Since a simple scaling of the elastic results for deducing the actual inelastic stress magnitudes everywhere in the model is difficult, let us see whether this can be achieved at a smaller scale. For this purpose triangular markers were placed in Figure 6 and Figure 7 about $7.5 \mathrm{~m}$ above the stope backs, which corresponds to about half the strike length of the stopes - yellow markers are above the primary stopes, whereas magenta markers are above the trailing secondary blocks. 

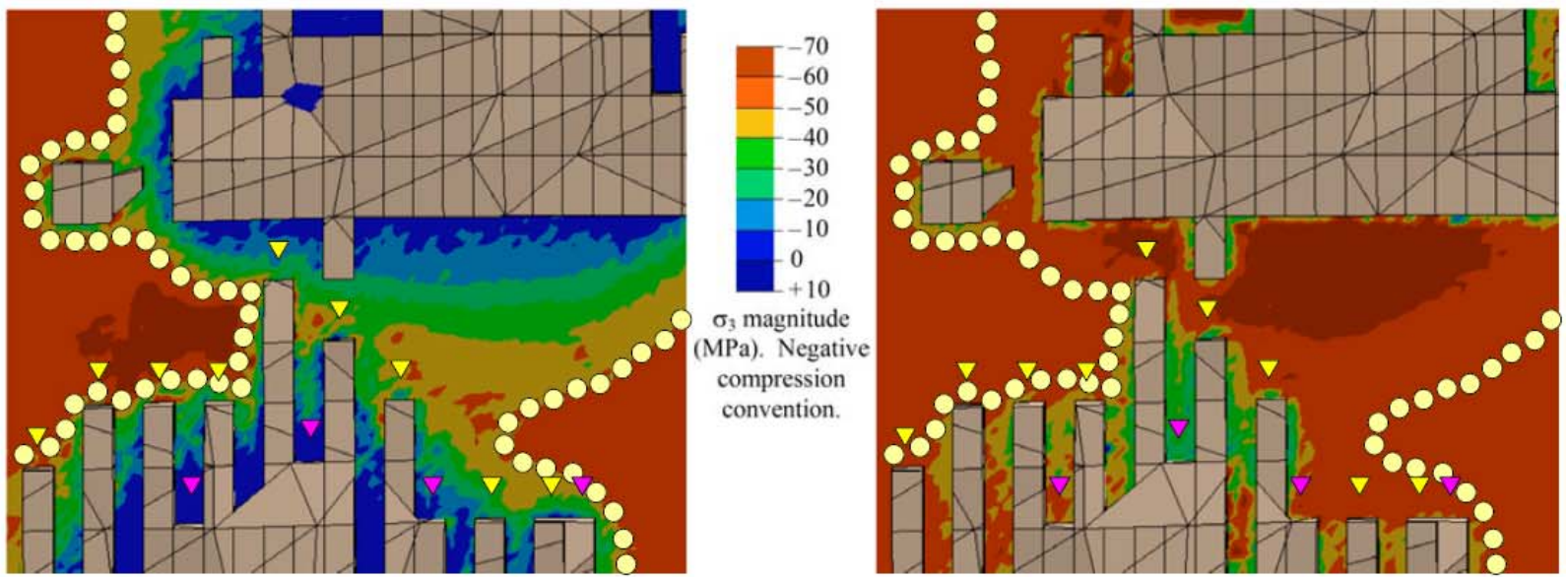

Inelastic strain-softening.

(a) At mining step A.

Elastic.
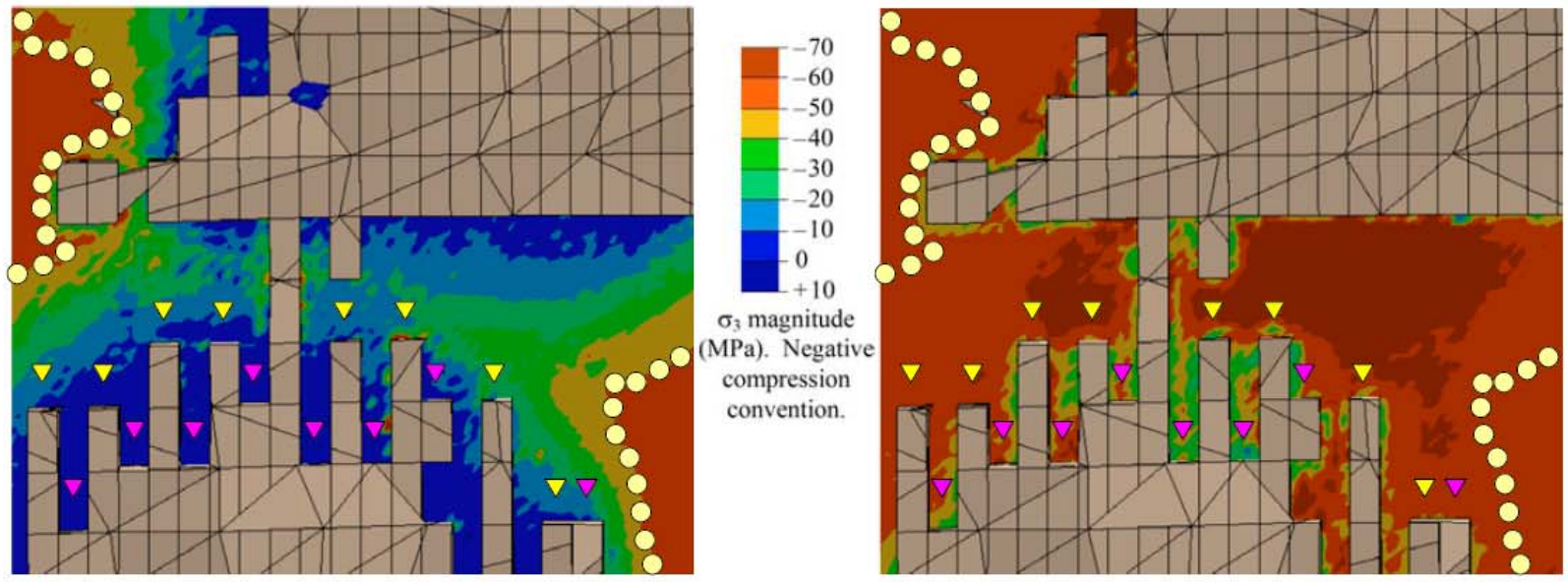

Inelastic strain-softening.

(b) At mining step $B$.

Elastic.

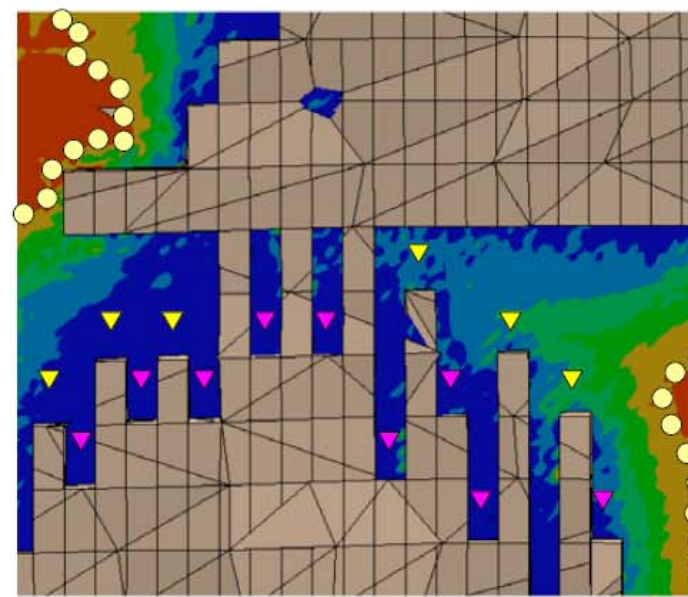

Inelastic strain-softening.

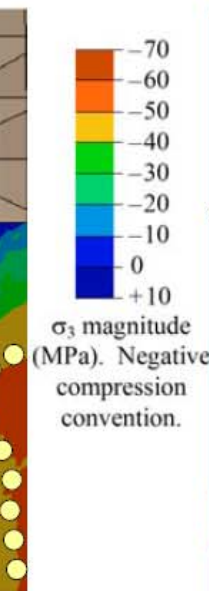

(c) At mining step $C$.

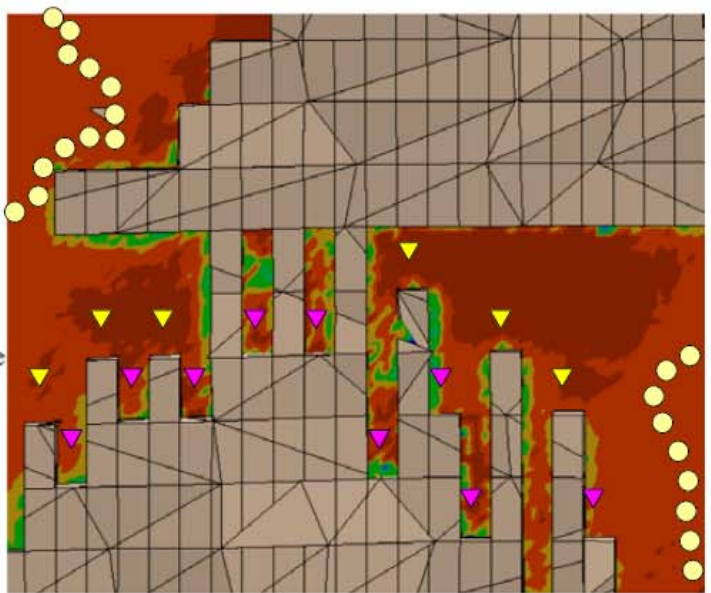

Elastic.

Figure 7 Elevation views showing the evolution of the minor principal stress magnitude ahead of the same mining front and at the same mining steps as in Figure 6, for the same inelastic strain-softening (left) and elastic (right) rock mass behaviours

Figure 8 compares the strain-softening and elastic $\sigma_{1}$ and $\sigma_{3}$ magnitudes at these locations above the stopes for all three mining steps. If consistent trends existed between the elastic and inelastic results, which could be used to "convert" the former to the latter, then elastic results could be used to reliably infer the state of failure at the control points. Although a few trends can be identified between the elastic and inelastic results, 
as shown in Figure 8a, for example, there is generally no consistent relationship between the two, and, more importantly, no relationship that holds true over multiple mining steps.

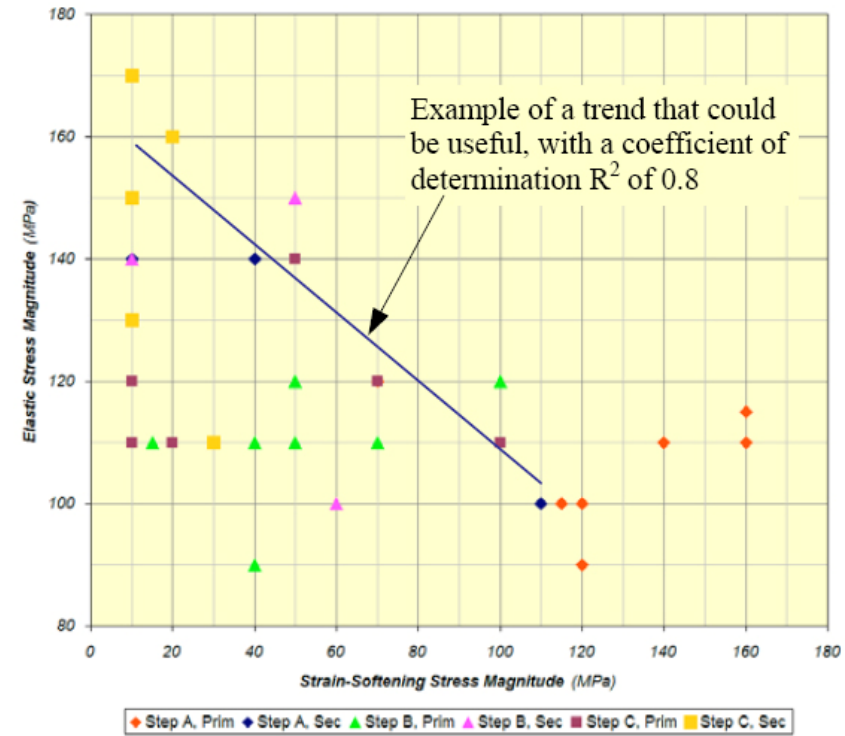

(a) $\sigma_{1}$ magnitude comparison.

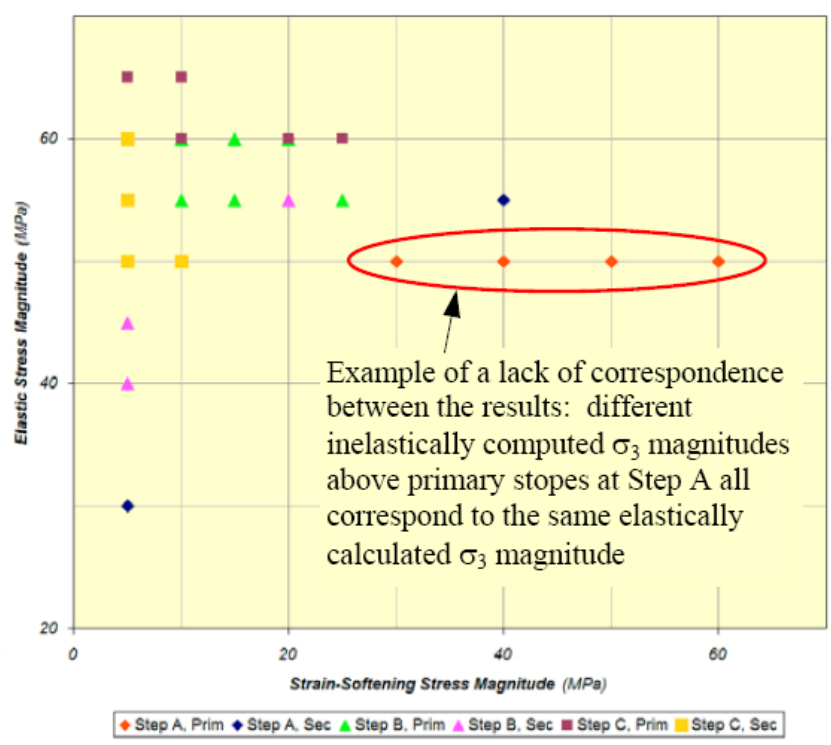

(b) $\sigma_{3}$ magnitude comparison.

Figure 8 Comparison of the $\sigma_{1}$ (a) and $\sigma_{3}(\mathrm{~b})$ magnitudes for the strain-softening and elastic results

This can make it difficult to derive, beforehand (i.e. for forward analyses), a single criterion to interpret elastic results in the case of highly stressed shrinking pillars between converging mining fronts. In other words, although it is possible to match elastically computed stresses with past field observations, it is much more difficult to establish, a priori, a unique criterion that will remain valid throughout the extraction of a highly stressed pillar.

Interpreting elastic results can be even more difficult in situations where extensive failure occurs in an "exponential" manner, as can be the case, for example, when very highly stressed mining fronts converge. In this instance, the failed regions in the pillar between the fronts grow faster with successive mining steps since, as mining progresses, this pillar becomes smaller and loses confinement, and, hence, strength as well. This, combined with the strain-softening behaviour (which very much "drives" failure, as discussed in the context of Figure 3) typical of the moderately jointed hard rock masses often encountered in underground metal mines, results in ever larger zones of failure, and ever larger stress transfers being required in order to reach equilibrium after successive stopes are removed. A particular difficulty associated with attempting to interpret elastic results in these circumstances is that any empirically-developed criterion tends to change as the mining sequence progresses.

\subsubsection{Centre of gravity of effort for inelastic modelling}

Contrary to the elastic approach, whereby relatively few parameters are required prior to launching analyses but results may be arduous to interpret, much information - not easily obtainable - is required before any inelastic simulation can be initiated. Deriving, adjusting and validating this information constitutes the bulk of the effort associated with inelastic modelling. Let us assume that a strain-softening behaviour is selected, along with the linear Mohr-Coulomb failure criterion shown in Figure 4a. In this case, the following input parameters are required at the scale of the rock mass for each of the geological horizons considered in the model, prior to any simulation being launched:

- The density of the material, as well as its Young's modulus and Poisson's ratio (similarly to what is required for elastic modelling).

- The peak and residual values of cohesion, tensile strength and friction angle (as shown in Figure 5). 
- The critical strain intervals (the amount of cumulative irreversible plastic strain between peak strength [taken to correspond to the end of the elastic response] and residual strength) associated with the cohesion, tensile strength and friction angle.

Usually, a first-pass assessment of the peak cohesion, tensile strength and friction angle values is obtained using the methodology described by Hoek et al. (1995), based upon the UCS of the intact material, its $\mathrm{m}_{\mathrm{i}}$ value and the GSI of the rock mass (GSI being often approximated as RMR89-5, with RMR89 being as per Bieniawski [1989]). Back-analyses of previous occurrences of instability are then done to refine these values, as well as derive the residual values of cohesion, tensile strength and friction angle, and their critical strain intervals. Large recorded seismic events and significant episodes of stress cycles can be used for this purpose, as described by Andrieux et al. (2004). A powerful approach has been recently derived that uses thousands of recorded seismic events, when available, to adjust residual strength values and critical strain intervals by matching the failure front computed by the model at successive mining steps with the seismicity recorded during the corresponding time intervals (Andrieux et al., 2008, in preparation).

Note that if a discrete element code is implemented, then the mechanical properties of the interfaces between the elements in the model (i.e. the faults, joint sets, contacts between the geological units, etc.) need to also be taken into account, including an adequate failure criterion and the appropriate post-peak properties for each type of discontinuity. Often, the "area contact elastic/plastic with Coulomb slip failure" joint constitutive model is retained, whereby failure in shear or tension results in frictional only behaviour following failure - with this approach, any initial cohesion and tensile strength is brought to zero at failure, with the friction angle value being maintained.

As can be seen from the previous discussion, inelastic numerical modelling, when complex constitutive behaviours are retained and multiple geological horizons and discontinuities are considered, is not a trivial affair. However, experienced modellers can generally derive (from back-analyses) input parameters that, although not perfect, are accurate enough to provide valid future predictions without the need to go through an extensive and sometimes nebulous "post-computation interpretation" phase.

\section{When is an elastic approach adequate with deep and high stress mining?}

In certain instances deep and highly stressed mining fronts can be modelled with an elastic code. When the situation involves a very strong and highly confined rock mass in which narrow stopes are extracted - a situation whereby failure in the rock mass ahead of mining is limited to the immediate abutment of the excavations - elastic numerical modelling can give results that are generally comparable to those inelastic modelling would give. Figure 9 shows an example for a deep and highly stressed metal mine in Canada.

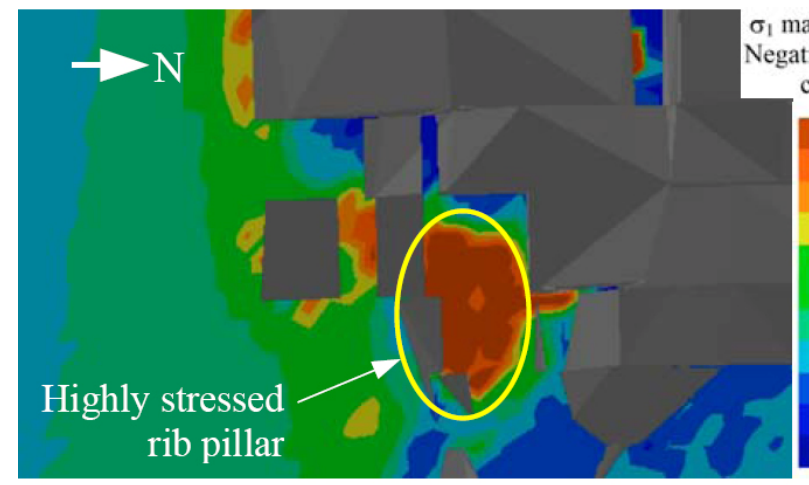

(a) Inelastic strain-softening.

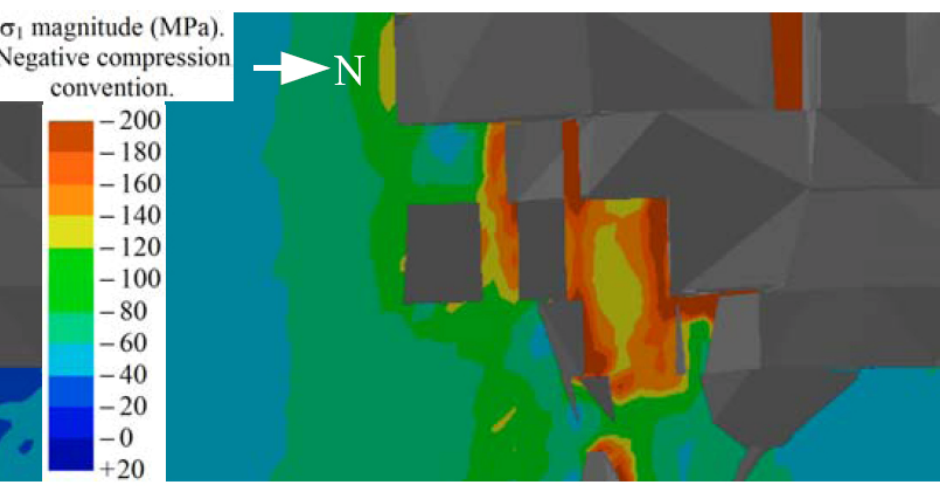

(b) Elastic.

Figure 9 Elevation views showing the magnitude of the major principal stress around excavations, for an inelastic strain-softening (a) versus elastic (b) rock mass behaviour. Example where the elastic modelling is showing some concordance with the inelastic results

In this example the modelling exercise (performed with 3DEC) was focusing on a region located in the abutment of the mine at a depth of about $1000 \mathrm{~m}$, where the geology comprised very strong massive 
sulphides (with a UCS of over $200 \mathrm{MPa}$ ) with few discontinuities. The ore lens in this area where the orebody tapers off is quite narrow, which results in narrow stopes (in the hanging wall-to-footwall direction) being excavated. Due to production constraints a stoping block had to be taken out of sequence, which resulted in the creation of a highly-stressed rib pillar (highlighted in Figure 9a).

Comparing the stress magnitudes computed by the two models shows that, although the actual stress magnitudes are different, very high levels are predicted by both approaches on the south edge of the pillar, along the abutment of the out-of-sequence block. As a result, both approaches would largely agree in predicting difficult mining conditions under very high stress levels. Note however that this agreement occurs in this case only in one area of the model, with significant differences being observed in other regions, where failure of the rock mass is predicted by the inelastic approach to have occurred.

\section{When is an inelastic approach necessary with deep and high stress mining?}

It is difficult to avoid implementing an inelastic numerical modelling approach in situations where significant rock mass failure is known (or expected) to occur ahead of and around mining. Section 2.3.1 has described the difficulties that are encountered when an elastic method is used under such circumstances. As discussed, using an elastic approach in a case where significant failure does occur requires extensive interpretation of the results in order for practical recommendations to be formulated. This exercise becomes particularly difficult when numerous pillars are involved where the ground stresses can migrate, and where the pillars' individual behaviour is controlled by their current state of failure and remaining strength. In this case it can become extremely difficult to "interpret", based solely upon elastic results, where the loads will transfer and how much each pillar will contribute to the load bearing capacity of the system. The discussion presented in Section 2.3.1 around Figure 6 and Figure 7 shows an example where the elastic approach would have suffered serious limitations.

Figure 10 shows an interesting case where the inelastic and elastic approaches produced very different - almost "opposite" - answers. In this example the analyses focused on the recovery of a highly stressed sill pillar between two converging mining fronts in a deep metal mine in Ontario, the lower front progressing upwards towards the upper one. The figure shows elevation views of principal stress vectors colour-coded by $\sigma_{1}$ magnitude for an inelastic strain-softening versus an elastic rock mass behaviour. Note that due to the non-planarity of the orebody some out-of-plane effects are visible on the figures (no single viewing plane could be cut that remained inside the orebody over the full height of the views shown).

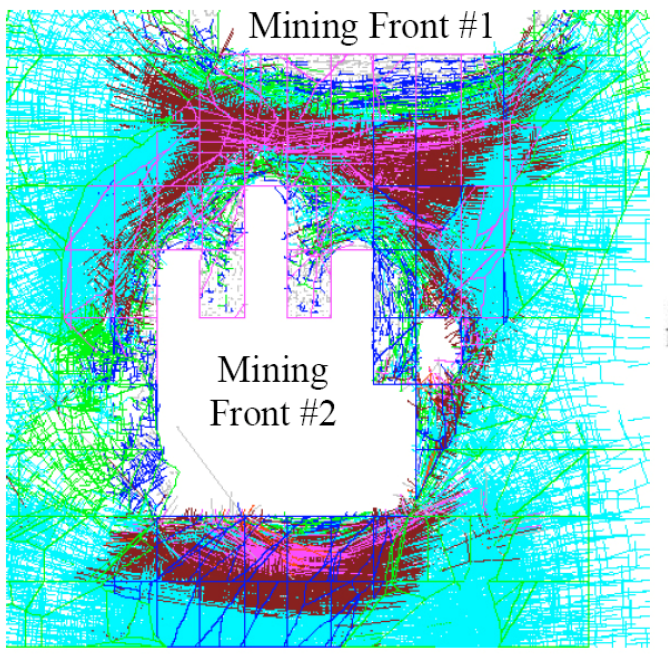

(a) Inelastic strain-softening.

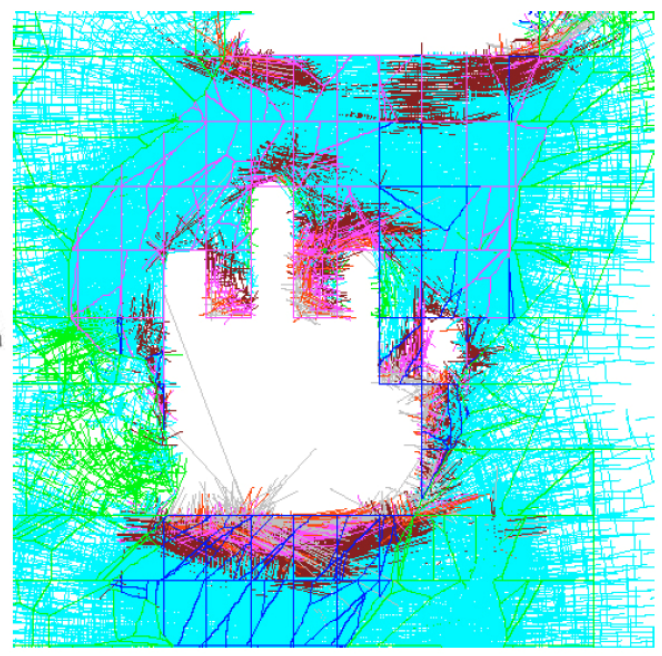

(b) Elastic.

Figure 10 Elevation views showing 3DEC-inferred principal stress vectors colour-coded by $\sigma_{1}$ magnitude around excavations, for an inelastic strain-softening (a) versus elastic (b) rock mass behaviour 
Note where the high stress areas locate in each case: in the core of the sill pillar ahead of mining with the inelastic approach, versus in the immediate abutment of the excavations with the elastic formulation. Note also how different the computed stress levels are in the secondary stopes - these blocks are predicted by the inelastic approach to be largely failed and destressed, whereas the elastic method shows them highly stressed. The elastic results would, in this case, be highly misleading on the nature of the mining conditions to expect in the various stopes (and on how to address them) without a significant interpretation of the direct results (basically a full "inversion" of these results).

\section{Practical information obtainable from advanced failure analyses}

Practical information can be extracted from advanced failure analyses - the exclusive domain of inelastic techniques - such as determining the degree of failure of a rock mass and the expression of this failure, by examining the stress state of the failed numerical zones in the model for the purpose of assessing their position along the post-peak strain-softening response. This information can, in turn, provide valuable information on the likely behaviour of the rock mass.

Failure analyses start by identifying the centroid of each numerical zone in the model that has, at one point during the computation, encountered the failure envelope. Note that with a strain-softening behaviour encountering the failure envelope does not mean that the numerical element has "entirely failed", but, rather, that it has started its "journey" between peak strength and residual strength, as discussed in Section 2.2. In order to provide an indication of where these numerical zones stand between their peak and residual states, their centroid markers can be colour-coded by the magnitude of the stress field at their location. A high stress magnitude indicates conditions still close to peak (whereby only limited strain-softening has occurred), whereas a low stress magnitude generally indicates conditions close to residual (whereby extensive softening has occurred). Figure 11 shows an example of this approach, in the case of the numerical back-analysis (for calibration purposes) of a mining sequence in a deep Canadian metal mine.

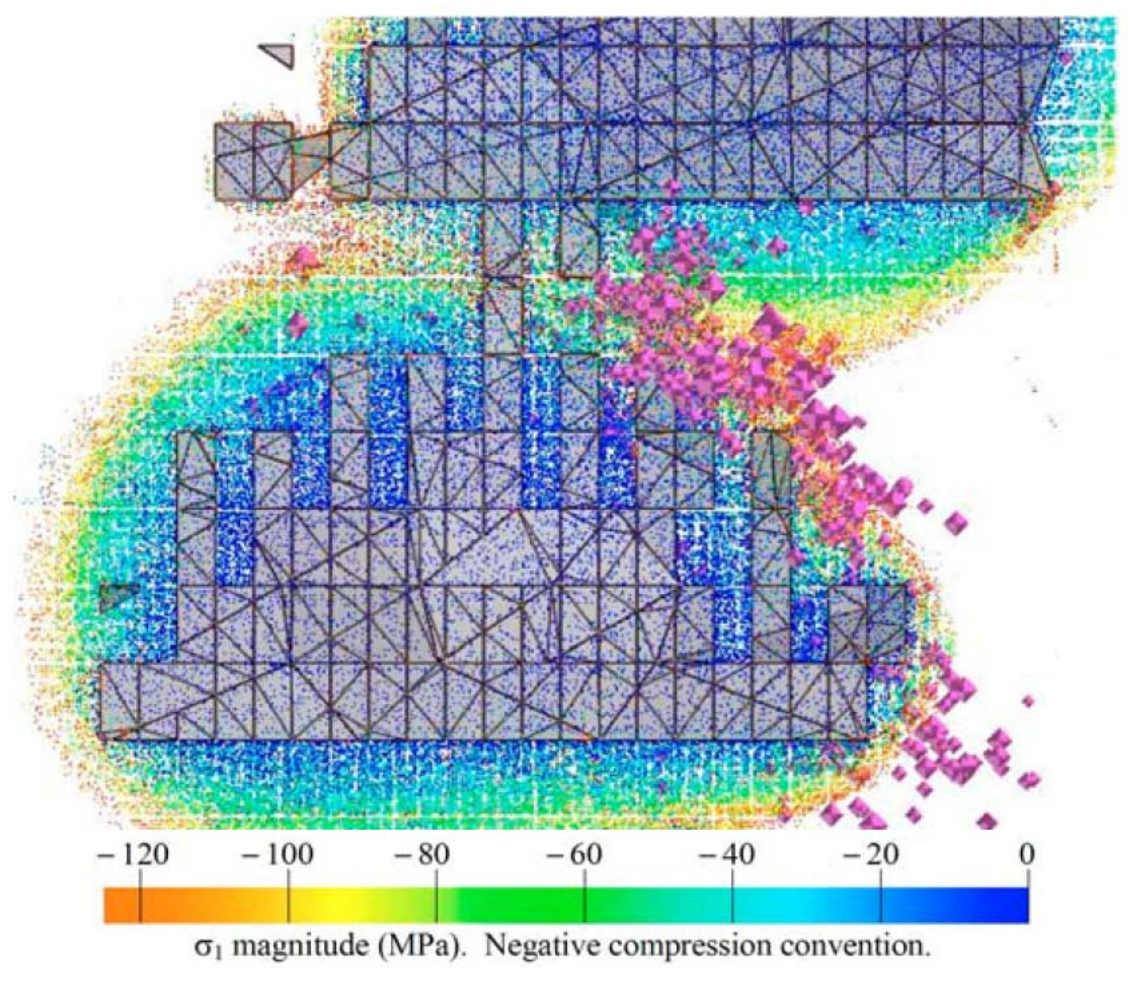

Figure 11 Elevation view showing 1) the centroid of each numerical element that has encountered the failure envelope during the simulations, colour-coded by its $\sigma_{1}$ magnitude, and 2) the seismicity recorded during the time step. Note that the failure indicators in the footwall and hanging wall have been removed from the view, for clarity. These strain-softening results were generated with the 3DEC code 
The dots in Figure 11 that are coloured orange and red identify numerical zones that, although having entered their post-peak phase, are still carrying loads over $100 \mathrm{MPa}$. On the other hand, the dots associated with varying shades of blue indicate numerical zones that carry little load (under $40 \mathrm{MPa}$ ). Note how well the location of the seismicity recorded during the corresponding time period matches the location of the failed elements still carrying high stresses (and, hence, still capable of releasing significant amounts of strain energy as failure progresses). Note, on the other hand, the absence of seismicity in regions completely failed (by now at their residual state).

This type of failure analysis can be used to estimate whether or not seismicity is likely to be associated with the stress-induced failure of the rock mass, and, if so, at which mining steps and where.

\section{Concluding remarks}

Although numerical modelling is by now a well accepted and widely used geomechanics tool, the applicability of the various types of formulation is not always well understood and still the subject of much debate. This paper has attempted to explain the basic differences between the elastic and inelastic approaches, as well as provide practical guidelines concerning which one to use for various sets of circumstances. In certain deep and highly stressed situations, whereby the failure front is located in the immediate vicinity of the excavations (i.e. does not extend very far inside the rock mass), the simpler elastic approach can provide results that are somewhat comparable to those produced by a more sophisticated inelastic formulation. In many instances, however, it can be very difficult to correctly interpret the results computed by an elastic approach when highly stressed mining fronts are involved and extensive failure occurs. In this case it may be more appropriate to implement a proper inelastic approach, even though this means having to go through the calibration effort required, and, subsequently, the much longer computer run times associated with inelastic simulations.

\section{References}

Andrieux, P., Hudyma, M., O’Connor, C., Li, H., Cotesta, L. and Brummer, R. (2008) (in preparation) Calibration of Large-Scale Three-Dimensional Non-Linear Numerical Models of Underground Mines Using Microseismicity Data. Proceedings $1^{\text {st }}$ International FLAC/DEM Symposium, Minneapolis, MN, USA, August 2008.

Andrieux, P., Zhu, H., Labrie, D., Doucet, C., Lampron, S. and Fleury, D. (2004) Determination and Validation of the Rock Mass Post-Peak Mechanical Properties for a 3DEC Strain-Softening Model of the 680 Sill Pillar at Louvicourt Mine. In Numerical Modelling of Discrete Materials in Geotechnical Engineering, Civil Engineering and Earth Science, H. Konietzky (editor), pp. 121-127. Proceedings $1^{\text {st }}$ International UDEC/3DEC Symposium, Bochum, Germany, September 2004. Lisse: Balkema, 2004.

Assonvi, C. and Richter, R. (1979) The Continuum Theory of Rock Mechanics. Series on Rock and Soil Mechanics, published by Trans Tech Publications, Inc., Zurich, Switzerland, 332 p.

Bieniawski, Z. (1989) Engineering Rock Mass Classifications: A Complete Manual for Engineers and Geologists in Mining, Civil, and Petroleum Engineering. John Wiley \& Sons publishers, New York, NY, USA, 272 p.

Hoek, E., Kaiser, P. and Bawden, W. (1995) Support of Underground Excavations in Hard Rock, Published by A.A. Balkema, Rotterdam, Netherlands, $215 \mathrm{p}$.

Hoek, E. and Brown, E.T. (1980) Underground Excavations in Rock. Published by the Institution of Mining and Metallurgy, 527 p, London, U.K.

Itasca (2003) 3DEC Theory and Background. Published by the Itasca Consulting Group, Minneapolis, MN, USA, $218 \mathrm{pp}$. 